\title{
A GENERALIZATION OF A THEOREM BY BOCHNER
}

\author{
DORTE OLESEN
}

\begin{abstract}
A theorem of Bochner states that if $\mu$ is a complex Borel measure on the $n$-dimensional torus $\mathbf{T}^{n}$ with Fourier-coefficients that vanish outside a proper cone in $\mathbf{Z}^{n}$, then $\mu$ is absolutely continuous with respect to Haar measure on $\mathbf{T}^{n}$. This result is generalized to a $C^{*}$-algebra setting using the concept of spectral subspaces for an $n$-parameter group of automorphisms and its dual group, in the case where the cone is the positive "octant".
\end{abstract}

Introduction. The classical F. and M. Riesz theorems state that a complex Borel measure on the unit circle $\mathbf{T}$ which is analytic, i.e. its negative Fouriercoefficients all vanish, is either identically zero or has the same null-sets as Haar measure.

In [2], S. Bochner showed that a measure on $\mathbf{T}^{n}$ with Fourier-coefficients that vanish outside a proper cone in $\mathbf{Z}^{n}$ is absolutely continuous with respect to Haar measure on $\mathbf{T}^{n}$.

In [4], F. Forelli regarded an action of the real line $\mathbf{R}$ as a topological transformation group on the locally compact Hausdorff space $S$. He defined a measure $\lambda$ to be analytic if its spectrum $\operatorname{sp}(\lambda)$ with respect to the action was nonnegative, and to be quasi-invariant if its collection of null-sets was carried into itself by the action of $\mathbf{R}$ on $S$. As a generalization of the F. and M. Riesz theorems he obtained the result that analytic measures are quasi-invariant. The notion of quasi-invariance was extended to the class of positive linear functionals on a $C^{*}$-algebra acted upon by a group of *-automorphisms in [5].

As a noncommutative generalization of Forelli's result, W. Arveson proved [1] that if a linear functional $\phi$ on a $C^{*}$-algebra had nonnegative spectrum with respect to a one-parameter group of *-automorphisms, the variation $|\phi|$ was quasi-invariant.

Here we show that Arveson's result can be used to prove quasi-invariance of functionals with spectrum in $[0, \infty)^{n}$, in the case of an $n$-parameter group action on a $C^{*}$-algebra. As a corollary we obtain that an analytic measure on $\mathbf{T}^{n}$ is either identically zero or has the same null-sets as Haar measure.

(For a short proof of Bochner's theorem in two dimensions, the reader may consult $[8,8.2 .5]$.)

I want to thank W. Arveson for encouraging my research in this field.

Received by the editors April 15, 1975.

AMS (MOS) subject classifications (1970). Primary 46L05; Secondary 43A05.

Key words and phrases. $C^{*}$-algebras, automorphism groups, spectral subspaces, quasi-invariant functionals.

- American Mathematical Society 1976 
Notation. In the following, $A$ denotes a $C^{*}$-algebra, $A^{\prime}$ its dual space. By a representation $(\alpha, A)$ of $\mathbf{R}^{n}$ we mean a homomorphism from $\mathbf{R}^{n}$ into the group of *-automorphisms of $A$ such that for every $x$ in $A$

$$
\left\|\alpha_{\left(t_{1}, \ldots, t_{n}\right)} x-x\right\| \rightarrow 0 \text { as }\left(t_{1}, \ldots, t_{n}\right) \rightarrow(0, \ldots, 0) .
$$

Let $\alpha(f) x$ with $f \in L^{1}\left(\mathbf{R}^{n}\right), x \in A$ denote the vector-valued integral

$$
\alpha(f) x=\int_{\mathbf{R}^{n}} f\left(t_{1}, \ldots, t_{n}\right) \alpha_{\left(t_{1}, \ldots, t_{n}\right)} x d t_{1} \cdots d t_{n} .
$$

Take $E \subseteq \mathbf{R}^{n}$. Define

$$
R^{\alpha}(E)=[\{\alpha(f) x \mid \operatorname{supp} \hat{f} \subset E, x \in A\}],
$$

[] denoting closed linear span,

$$
\hat{f}\left(s_{1}, \ldots, s_{n}\right)=\int_{\mathbf{R}^{n}} f\left(t_{1}, \ldots, t_{n}\right) \exp \left(i \sum s_{k} t_{k}\right) d t_{1}, \ldots, d t_{n} .
$$

Define for a closed set $E \subseteq \mathbf{R}^{n}$

$$
M^{\alpha}(E)=\left\{x \in A \mid \alpha(f) x=0 \forall f \in I_{0}(E)\right\}
$$

where $I_{0}(E)=\left\{f \in L^{1}\left(\mathbf{R}^{n}\right) \mid \hat{f}\right.$ vanishes on a neighbourhood of $\left.E\right\}$.

Let $\phi$ be a positive linear functional on $A$. Let $\left(\pi_{\phi}, H_{\phi}, \xi_{\phi}\right)$ denote the representation obtained from $\phi$ using the Gelfand-Naimark-Segal construction $[3,2.4 .4]$.

With $(\alpha, A)$ a representation of $\mathbf{R}^{n}$, the transposed action

$$
\left(\alpha_{t}^{\prime} \phi\right)(x)=\phi\left(\alpha_{-t} x\right) \quad \forall t \in \mathbf{R}^{n} \forall x \in A \forall \phi \in A^{\prime}
$$

defines a group of isometries of $A^{\prime}$ which takes positive functionals into positive functionals and satisfies that for $t$ in $\mathbf{R}^{n}$,

$$
\left|\alpha_{t}^{\prime} \phi(x)-\phi(x)\right| \rightarrow 0 \text { as } t \rightarrow 0 \forall x \in A .
$$

We say that $\phi$ in $A^{\prime}$ is continuous if in fact $\left\|\alpha_{t}^{\prime} \phi-\phi\right\| \rightarrow 0$ as $t \rightarrow 0$. We use $|\phi|$ to denote the absolute value or variation of $\phi$, obtained by polar decomposition $[3,12.2]$.

We call $\phi$ quasi-invariant if the representations $\pi_{|\phi|}$ and $\pi_{\alpha^{\prime}|\phi|}$ are quasiequivalent $[3,5.3]$.

When $T$ is a locally compact space, $C_{0}(T)$ denotes the continuous functions vanishing at infinity, and $M(T)$ the dual space of $C_{0}(T)$.

\section{The main theorem.}

THEOREM. Let $(\alpha, A)$ be a representation of $\mathbf{R}^{n}$. Given a coordinate system, let $V=[0, \infty)^{n}$, and let $C V$ denote the complement of $V$. If $\phi$ is a bounded linear functional on $A$ which for some $s=\left(s_{1}, \ldots, s_{n}\right)$ in $\mathbf{R}^{n}$ satisfies the equivalent conditions

(i) $\phi$ annihilates $R^{\alpha}(s-C V)$,

(ii) $\phi \in M^{\alpha^{\prime}}(-s+V)$,

then $|\phi|$ is quasi-invariant and continuous. 
Proof. That (i) and (ii) are equivalent is proved in [7, Proposition 2.3.4(iii)] as a consequence of the duality relation $\left(\alpha_{t}^{\prime} \phi\right)(x)=\phi\left(\alpha_{-t} x\right)$. Condition (i) entails that $\phi$ annihilates each $R^{\alpha^{i}}\left(s_{i}, \infty\right), \alpha_{t_{i}}^{i}$ denoting the one-parameter subgroup $\alpha_{\left(0, \ldots, 0, t_{i}, 0, \ldots, 0\right)}$, since

$$
s-C V=\left(s_{1}, \ldots, s_{n}\right)-C V=\bigcup_{i=1}^{n}\left(s_{i}, \infty\right) \underset{j \neq i}{\times} \mathbf{R}_{j}
$$

and so by [7, Proposition 2.3.4(ii) and Lemma 2.4.8(iv)], $R^{\alpha}(s-C V)$ is the closed linear span of spaces $R^{\alpha^{i}}\left(s_{i}, \infty\right)$. For brevity, let $(\pi, H, \xi)$ denote the representation obtained from $|\phi|$ using the Gelfand-Naimark-Segal construction. By the generalization to $C^{*}$-algebras of the $\mathrm{F}$. and $\mathrm{M}$. Riesz theorems [1, Theorem 5.3], $|\phi|$ is then covariant with respect to each subgroup $\alpha^{i}$, i.e. there exist strongly continuous one-parameter unitary groups $u^{i}$ in $B(H)$ such that

$$
\pi\left(\alpha_{t_{i}}^{i}(x)\right)=u_{t_{i}}^{i} \pi(x) u_{-t_{i}}^{i} \quad \forall t_{i} \in \mathbf{R} \forall x \in A .
$$

It follows that

$$
|\phi|\left(\alpha_{\left(t_{1}, \ldots, t_{n}\right)} x\right)=\left(\pi(x) u_{-t_{n}}^{n} \cdots u_{-t_{1}}^{1} \xi \mid u_{-t_{n}}^{n} \cdots u_{-t_{1}}^{1} \xi\right) .
$$

This shows that $\pi$ and $\pi_{\alpha^{\prime}|\phi|}$ are unitarily equivalent; thus $|\phi|$ is quasi-invariant. Furthermore

$$
\begin{aligned}
\left(\alpha_{t_{i}}^{i}|\phi|-|\phi|\right)(x) & =\left(\pi(x) u_{t_{i}}^{i} \xi \mid u_{t_{i}}^{i} \xi\right)-(\pi(x) \xi \mid \xi) \\
& =\left(\pi(x)\left(u_{t_{i}}^{i} \xi-\xi\right) \mid u_{t_{i}}^{i} \xi\right)+\left(\pi(x) \xi \mid u_{t_{i}}^{i} \xi-\xi\right)
\end{aligned}
$$

implies that

$$
\left\|\left(\alpha_{t_{i}}^{i}\right)^{\prime}|\phi|-|\phi|\right\| \leqslant 2\left\|u_{t_{i}}^{i} \xi-\xi\right\|
$$

thus

$$
\left\|\left(\alpha_{t}\right)^{\prime}|\phi|-|\phi|\right\| \rightarrow 0 \text { as } t=\left(t_{1}, \ldots, t_{n}\right) \rightarrow 0 .
$$

REMARK. If the cyclic vector $\xi$ is also separating for the weak closure $\pi(A)^{\prime \prime}$ of $\pi(A)$ on $H$, then quasi-invariance of $|\phi|$ implies covariance, i.e. the existence of a strongly continuous unitary group in $B(H)$ such that for every $x$ in $A$ and $\left(t_{1}, \ldots, t_{n}\right)$ in $\mathbf{R}^{n}$

$$
\pi\left(\alpha_{\left(t_{1}, \ldots, t_{n}\right)}(x)\right)=u_{\left(t_{1}, \ldots, t_{n}\right)} \pi(x) u_{\left(-t_{1}, \ldots,-t_{n}\right)} .
$$

To see this, note that quasi-invariance means that the automorphism group on $A$ carries over to $\pi(A)^{\prime \prime}$. By [6, Corollary 3.6] a weakly continuous group of automorphisms on a von Neumann algebra has a strongly continuous unitary implementation whenever the group is locally compact and the algebra has a cyclic and separating vector.

It follows that if $A$ is commutative, quasi-invariance and covariance are identical notions.

Thus it is an immediate consequence of the Theorem above that we have the following 
Corollary. Let $T$ be a locally compact Hausdorff space, $\left(\alpha, C_{0}(T)\right)$ a representation of $\mathbf{R}^{n}$. Let $\mu$ in $M(T)$ for some $s=\left(s_{1}, \ldots, s_{n}\right)$ in $\mathbf{R}^{n}$ satisfy the equivalent conditions $\left(V=[0, \infty)^{n}\right.$ in some coordinate system):

(i) $\mu$ annihilates $R^{\alpha}(s-C V)$,

(ii) $\mu \in M^{\alpha^{\prime}}(-s+V)$.

Then the total variation $|\mu|$ of $\mu$ is covariant.

To relate this to the classical setting for the theorem of Bochner, let $\mathbf{T}^{n}$ denote the $n$-dimensional torus. Define an $n$-parameter group of ${ }^{*}$-automorphisms $\alpha_{\left(t_{1}, \ldots, t_{n}\right)}$ of $C\left(\mathbf{T}^{n}\right)$ by

$$
\left.\left(\alpha_{\left(t_{1}, \ldots, t_{n}\right)} f\right)\left(e^{i x_{1}}, \ldots, e^{i x_{n}}\right)=f^{i\left(x_{1}-t_{1}\right)}, \ldots, e^{i\left(x_{n}-t_{n}\right)}\right) .
$$

Let $\mu$ be a complex Borel measure on $\mathbf{T}^{n}$. It is seen using the density of trigonometric polynomials in $C\left(\mathbf{T}^{n}\right)$ that $\mu$ annihilates all $f$ in $R^{\alpha}\left(\left(s_{1}, \ldots, s_{n}\right)\right.$ $-C V)$ if and only if $\hat{\mu}$ has support in $\left(-s_{1}, \ldots,-s_{n}\right)+V$. Thus supp $\hat{\mu} \subset V$ implies that $\mu$ annihilates $R^{\alpha}(-C V)$ and we have that $|\mu|$ is covariant. So $|\mu|$ is either identically 0 or mutually equivalent to Haar measure on $\mathbf{T}^{n}$.

\section{REFERENCES}

1. W. Arveson, On groups of automorphisms of operator algebras, J. Functional Analysis 15 (1974), 217-243.

2. S. Bochner, Boundary values of analytic functions in several variables and of almost periodic functions, Ann. of Math. (2) 45 (1944), 708-722. MR 6, 124.

3. J. Dixmier, Les $C^{*}$-algèbres et leurs représentations, Cahiers Scientifiques, fasc. 29, GauthierVillars, Paris, 1964. MR 29 \#485.

4. F. Forelli, Analytic and quasi-invariant measures, Acta Math. 118 (1967), 33-59. MR 35 \#667.

5. A. Guichardet et $\mathrm{D}$. Kastler, Désintégration des états quasi-invariants des $C^{*}$-algébres, J. Math. Pures Appl. (9) 49 (1970), 349-380. MR 43 \#2524.

6. U. Haagerup, The standard form of von Neumann algebras, Math. Scand. 37 (1975), 271-283.

7. D. Olesen, On spectral subspaces and their applications to automorphism groups, (Proc. Meeting "Algebre $C^{*}$ e loro applicazioni fisica teorica," Rome, March 1975) Luminy Lectures 1973-74-Marseille CNRS Preprint 74/P602 (to appear).

8. W. Rudin, Fourier analysis on groups, Interscience Tracts in Pure and Appl. Math., no. 12, Interscience, New York, 1962. MR 27 \#2808.

Matematisk Institut, Odense Universitet, Niels Bohrs Alle, DK-5000 Odense, Denmark

Current address: Matematisk Institut, Universitetsparken 5, DK-2100 Copenhagen Ø, Denmark 